\title{
Sexual Function of 26-36-year-old Men With Type 1 Diabetes
}

Indrè Matulevičiūtè, Valentinas Matulevičius, Rytas Ostrauskas, Ilona Banišauskaitė, Justina Jurevičiūtė, Silvijus Abramavičius, Rasa Verkauskienè, Vaidotas Urbanavičius*

Lithuanian University of Health Sciences

Vilnius University*

Introduction. Sexual function of type 1 diabetic (T1D) males vs. controls has been scarcely investigated. Investigation of reproductive health of young males was performed in 2003/2004 ("The Reproductive Function of Estonian, Latvian and Lithuanian Young Men" (ELLY)) (1) in the Baltic countries although questions regarding sexual function were not addressed specifically at that time. ELLY study participants were invited as controls for investigation of sexual function in T1D males.

European Male Ageing Study (EMAS) Sexual Functioning Questionnaire (SFQ) (2) was used for the evaluation of sexual function.
Conclusion. As results of EMAS-SFQ show, changes of domains of sexual function appear progressively in 3 out of 4 sexual domains in the step forward fashion (masturbation-sexual functioning distressoverall sexual function) and are all finally found at 10-19 years of T1D duration.
Results. EMAS-SFQ: ELLY vs. various T1D durations.

\begin{tabular}{|c|c|c|c|c|}
\hline & OSF (mean; SD) & M (mean; SD) & SFD (mean; SD) & CSF (mean; SD) \\
\hline ELLY, N=80 & 21,$20 ; 5,62$ & 2,$27 ; 1,94$ & 1,$42 ; 1,93$ & $-0,36 ; 1,29$ \\
\hline T1D $0-4, N=14$ & 19,$50 ; 6,70$ & 0,$71 ; 0,82$ & 3,$86 ; 4,68$ & $-1,14 ; 2,71$ \\
\hline $\mathrm{p}$ & $>0,05$ & $<0,004$ & $>0,05$ & $>0,05$ \\
\hline T1D 5-9, N=19 & 18,$32 ; 7,02$ & 1,$56 ; 1,61$ & 3,$68 ; 3,77$ & $-0,47 ; 1,80$ \\
\hline $\mathrm{p}$ & $>0,05$ & $>0,05$ & $<0,0001$ & $>0,05$ \\
\hline T1D $0-9, N=33$ & 18,$82 ; 6,80$ & 1,$19 ; 1,37$ & 3,$76 ; 4,11$ & $-0,76 ; 2,22$ \\
\hline $\mathrm{p}$ & $>0,05$ & $<0,001$ & $<0,0001$ & $>0,05$ \\
\hline T1D $10-19, N=45$ & 19,$24 ; 5,34$ & 1,$27 ; 1,71$ & 3,$53 ; 4,70$ & $-1,0 ; 2,82$ \\
\hline $\mathrm{p}$ & $<0,026$ & $<0,003$ & $<0,026$ & $>0,05$ \\
\hline T1D $20-29, N=39$ & 17,$73 ; 7,26$ & 1,$25 ; 1,64$ & 3,$78 ; 4,38$ & $-0,97 ; 1,88$ \\
\hline $\mathrm{p}$ & $<0,01$ & $<0,003$ & $<0,003$ & $>0,05$ \\
\hline T1D $0-33, N=122$ & 18,$43 ; 6,51$ & 1,$29 ; 1,62$ & 3,$73 ; 4,36$ & $-0,98 ; 2,39$ \\
\hline $\mathrm{p}$ & $<0,003$ & $<0,0001$ & $<0,0001$ & $>0,05$ \\
\hline
\end{tabular}

References:

1. Grigorova M, Punab M, Zilaitienė B, Erenpreiss J, Ausmees K, Matulevičius V et al. . Genetically determined dosage of folliclestimulating hormone (FSH) affects male reproductive parameters. J Clin Endocrinol Metab. 2011 Sep;96(9):E1534-41

2. O'Connor DB, Corona G, Forti G, Tajar A, Lee DM, Finn JD, Bartfai G, Boonen S, Casanueva FF, Giwercman A, Huhtaniemi IT, Kula K, O'Neill TW, Pendleton N, Punab M, Silman AJ, Vanderschueren D, Wu FC. Assessment of sexual health in aging men in Europe: development and validation of the European Male Ageing Study sexual function questionnaire. J Sex Med 2008;5:1374-85.

3. Gerressu M, Mercer CH, Graham CA, Wellings K, Johnson AM. Prevalence of masturbation and associated factors in British national probability survey. Arch Sex Behav. 2008 Apr;37(2):266-78.

\section{Methods}

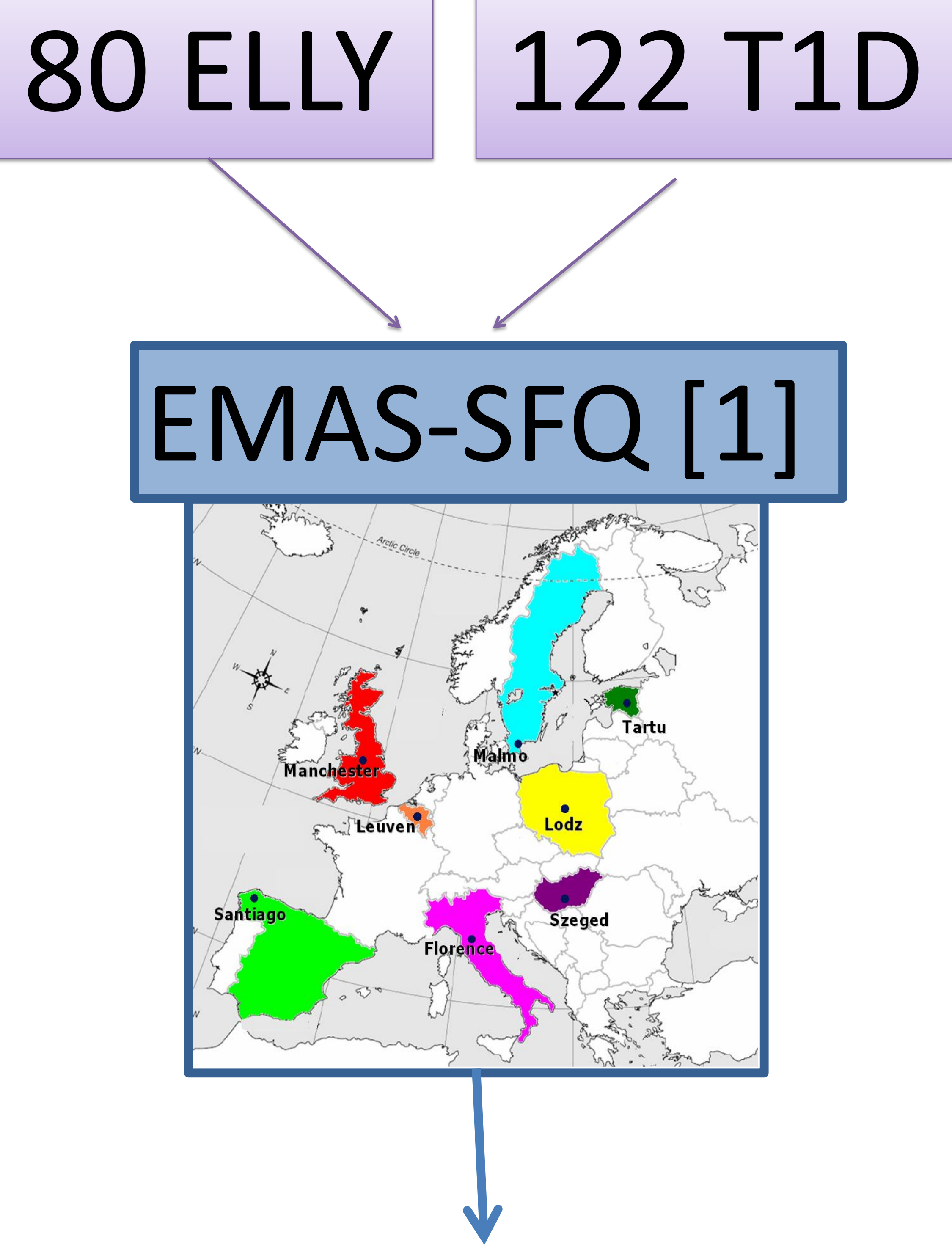

Masturbation (M) Q no. 7 (3)

Overall sexual function (OSF) Q no. $1,5,6,13,17$

Changes of sexual function during 1 year (CSF) Q no. 4,9,12,16,19

Sexual function distress (SFD)

Q no. $3,8,11,15,18$ 